\title{
Chemical synthesis and investigation of the native form and an improved gamma-core analogue of Neosartorya fischeri antifungal protein 2 (NFAP2)
}

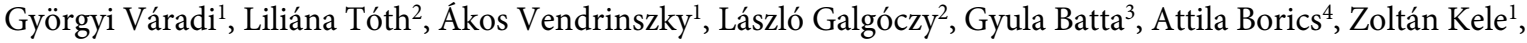 \\ Gábor K. Tóth ${ }^{1}$ \\ ${ }^{1}$ Departmentof Medical Chemistry, University of Szeged, Hungary \\ ${ }^{2}$ Departmentof Microbiology, Faculty of Science and Informatics, University of Szeged, Hungary \\ ${ }^{3}$ Department of Organic Chemistry, University of Debrecen, Hungary \\ ${ }^{4}$ Institute of Biochemistry, Biological Research Centre, Hungarian Academy of Sciences, Hungary
}

https://doi.org/10.17952/35EPS.2018.246

\section{Introduction}

NFAP2 is a novel cysteine-rich antifungal protein from Neosartorya fischeri N RRR 181 [ 1]. The Minimum Inhibitory Concentration(MIC) values on clinically relevant Candida species vary between 0.391 and $1.563 \mu \mathrm{g} / \mathrm{mL}$. NFAP2 is a cysteine-rich, cationic 52 -mer protein stabilized by three disulfide bridges. Based on homology to other cysteine-rich antifungal proteins, the $a b c a b c$ topology is feasible (Fig. 1).

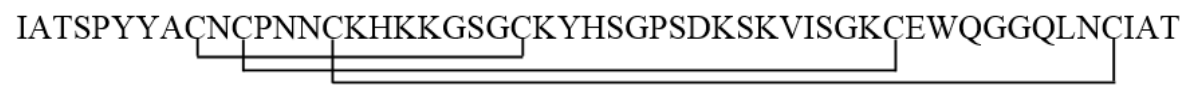

Figure 1: Sequence and supposed disulfide bond pattern of NFAP2

The aims of this study were the chemical synthesis and functional mapping of NFAP2. In order to improve the antifungal activity, the evolutionary conserved $\gamma$-core motif was modified, and an analogue of NFAP2 having a more active $\gamma$-core was synthesized. All these efforts can lead to a better understanding of the importance of $\gamma$-core and other parts of the protein on the antifungal effect.

\section{Results and Discussion}

NFAP2 was prepared by native chemical ligation of the $\mathrm{N}$-terminal22-mer thioester fragment and the C-terminal 30-mer part [2]. The thioester was synthesized on the previously described Cys-SH resin [3]. Both peptides were prepared on the solid-phase using Boc chemistry and DCC/HOBt coupling. Native chemical ligation of the purified fragments was conducted in a $\mathrm{pH} 7.5$ ammonium acetate buffer in the presence of $3 \%$ thiophenol for 3 hours. Oxidation of the thiols of cysteines was carried out in a glutathione redox buffer containing $1 \mathrm{mM}$ GSH and $1 \mathrm{mM}$ GSSG at pH 7.5 for 24 hours. Synthetic NFAP2 (sNFAP2) was compared to the native protein isolated from the ferment of Neosartorya fischeri N RRR 181 (nNFAP2) and to recombinant NFAP2 which was prepared by heterologous expression in a Penicillium chrysogenum based system (rNFAP2). RP-HPLC analysis, NMR and ECD spectroscopies, and antifungal susceptibility tests revealed the identity of synthetic, native and recombinant NFAP2.

For functional mapping, small fragments of NFAP2 were synthesized by solid-phase synthesis applying Fmoc chemistry and DCC/HOBt coupling. Surprisingly, not the highly conserved $\gamma$-core motif, but the mid-N-terminal part of the protein found to be functionally active. In contrary to the $\gamma$-core motif which is almost neutral (estimated charge at $\mathrm{pH} 7$ is -0.2) and slightly hydrophilic (GRAVY value is -0.993), the mid-N-terminal fragment is positively charged (estimated charge at $\mathrm{pH} 7$ is +3.1 ) and hydrophilic (GRAVY value is -1.682). Shuffling of amino acid sequence of the most effective fragment did not have effect on the antifungal activity. It proved the importance of physico-chemical properties and not the sequence on the antifungal effect.

The $\gamma$-core motif is a unifying structural signature in all classes of cysteine-rich antimicrobial peptides [4]. It is an evolutionary conserved region both in its amino acid sequence and its structure: two antiparallel $\beta$-strands are connected by a short turn region.

To examine the effect of $\gamma$-core on the antifungal activity, a 14-mer peptide (NFAP2 $\gamma$ ) and an improved analogue of it (NFAP2imp $\gamma$ ) were synthesized. While NFAP2 $\gamma$ did not inhibit the growth of the examined fungi (Saccharomyces cerevisiae SZMC 0644, Candida albicans ATCC 10231 and Candida parapsilosis CBS 604), 
the more positively-charged improved analogue (NFAP2imp $\gamma$ ) showed some antifungal effect.

An analogue of NFAP2 protein containing the peptide NFAP2imp $\gamma$ as $\gamma$-core was synthesized by native chemical ligation. Two methods were tried for the formation of disulfide bridges: air oxidation and the above mentioned glutathione redox buffer. According to our experience, either an oxidizing agent alone (e.g. $\mathrm{O} 2$ of air) or a redox system (e.g. GSH-GSSG buffer) can form the right pairing of cysteines in a protein. Surprisingly, both methods led to the same disulfide bond pattern in this case (Fig. 2). The major product was isolated from the reaction mixtures and was subjected to structural and biological investigations. NMR and ECD conformational studies showed an unordered structure for the protein, and microdilution test revealed weaker antifungal activity of the improved $\gamma$-core analogue than that of the native NFAP2 protein.
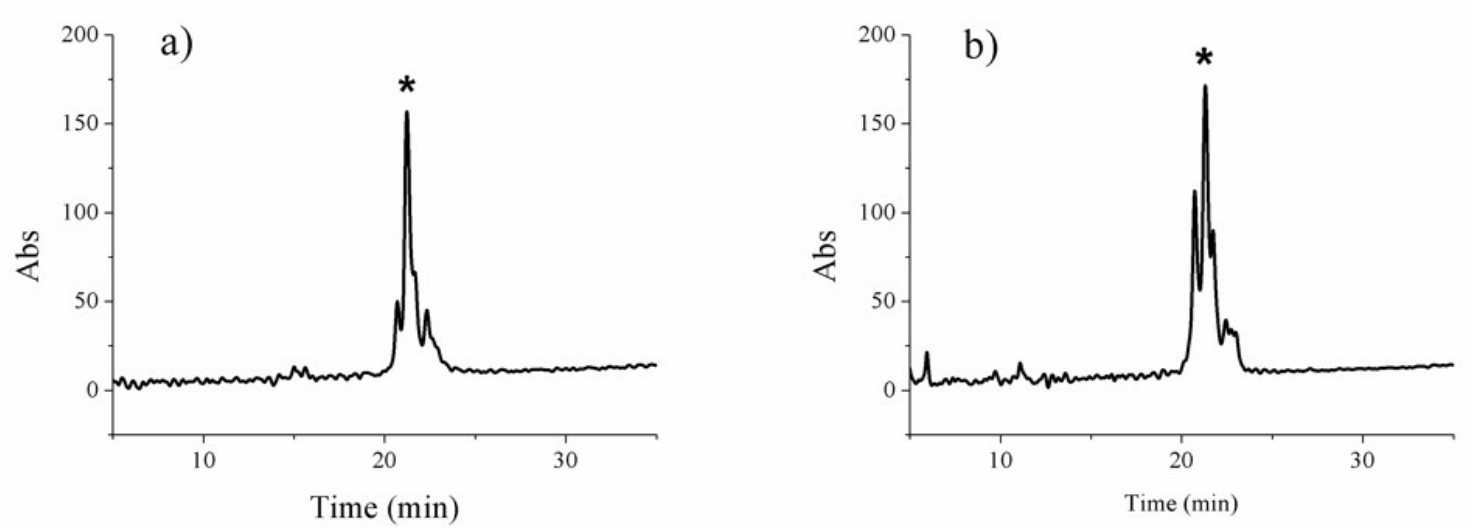

Figure 2: HPLC profiles of disulfide bond formation of the improved $\gamma$-core analogue of NFAP2 protein applying air oxidation (a) and a glutathione redox buffer $(b)$.

In conclusion, native chemical ligation followed by oxidation led to a protein that was proved to be identical with native NFAP2. Functional mapping revealed the mid-N-terminal part and not the $\gamma$-core as the most active fragment. Antifungal activity of the $\gamma$-core could be improved by increasing the number of positive charges and the hydrophilic character. It demonstrated the importance of physico-chemical properties on the antifungal effect. In contrary to our expectations, the improved $\gamma$-core-containing analogue of NFAP2 possessed lower antifungal activity than the native protein.

\section{Acknowledgements}

This work was supported by the EU and co-financed by the European Regional Development Fund under the project GINOP-2.3.2-15-2016-00014.

\section{References}

[1] Tóth, L., Kele, Z., Borics, A., Nagy, G. L., Váradi, G., Virágh, M., Takó, M., Vágvölgyi, C., Galgóczy, L., AMB Express, 2016, 6:75, doi:10.1186/s13568-016-0250-8.

[2] Dawson, P. E., Muir, T. W., Clarklewis, I., Kent, S. B. H., Science, 1994, 266, 776-779, doi:10.1126/ science.7973629.

[3] Váradi, G., Tóth, G. K., Kele, Z., Galgóczy, L., Fizil, Á., Batta, G., Chemistry - A European Journal, 2013, 19, 12684-12692, doi:10.1002/chem.201301098.

[4] Yount, N.Y. Yeaman, M.R., Proc. Natl. Acad. Sci. U.S.A., 2004, 101, 7363-7368, doi: 10.1073/ pnas.0401567101 